BRIEF REPORTS

\title{
Filling gaps in the distribution of the white-winged vampire bat, Diaemus youngii (Phyllostomidae, Desmodontinae): new records for southern Amazonia
}

\author{
Mônica A. PEDROSO ${ }^{*}$, Patrício A. da ROCHA², Marcus V. BRANDÃO ${ }^{3}$, Guilherme S. T. GARBINO ${ }^{4}$; \\ Carolina O. de MORAES 5 , Caroline C. AIRES ${ }^{5,6}$ \\ ' Universidade Federal de Sergipe, Programa de Pós-Graduação em Ecologia e Conservação, Campus São Cristóvão, CEP: 49100-00. Jardim Roza Elze, Sergipe, Brazil. \\ 2 Universidade Federal da Paraíba, Programa de Pós-Graduação em Zoologia, Campus I, Departamento de Sistemática e Ecologia, CEP: 58051-900. Castelo Branco, \\ João Pessoa, Paraíba, Brazil. \\ 3 Universidade Federal de São Carlos, Programa de Pós-Graduação em Diversidade Biológica e Conservação, Campus Sorocaba, CEP: 18052-780. Itinga, Sorocaba, \\ São Paulo, Brazil. \\ ${ }^{4}$ Universidade Federal de Minas Gerais, Programa de Pós-Graduação em Zoologia, Departamento de Zoologia, Instituto de Ciências Biológicas, CEP: $31270-901$. \\ Pampulha, Belo Horizonte, Minas Gerais, Brazil. \\ 5 Universidade de Mogi das Cruzes, Campus da Sede, CEP: 08780-911. Vila Partenio, Mogi das Cruzes, São Paulo, Brazil. \\ ${ }^{6}$ Universidade de São Paulo, Museu de Zoologia, Laboratório de Mastozoologia, CEP: 04299-970. Ipiranga, São Paulo, São Paulo, Brazil. \\ * Corresponding author: monicaapp@hotmail.com
}

\section{ABSTRACT}

Bats of the subfamily Desmodontinae are the only hematophagous mammals, represented by three species. Among them, Diaemus youngii has the fewest records in Brazil, being poorly known demographically and ecologically. We report the first record of D. youngii for Mato Grosso state, in central-western Brazil, and provide additional records for the states of Rondônia and Tocantins, in northern Brazil, extending the known distribution of D. youngii in the southern Amazon region.

KEYWORDS: rainforest, hematophagous bat, Mato Grosso, Rondônia, Tocantins

\section{Preenchendo lacunas na distribuição do morcego-vampiro de asas brancas, Diaemus youngii (Phyllostomidae, Desmodontinae): novos registros para o sul da Amazônia}

\section{RESUMO}

Os morcegos da subfamília Desmodontinae compreendem às únicas três espécies de mamíferos hematófagos. Entre elas, Diaemus youngii é a espécie com menor número de registros no Brasil, sendo pouco conhecida demográfica e ecologicamente. Nós relatamos o primeiro registro de D. youngii para o estado do Mato Grosso, no centro-oeste do Brasil, e fornecemos registros adicionais para os estados de Rondônia e Tocantins, no norte do Brasil. Os registros ampliam a distribuição conhecida de $D$. youngii no sul da regiáo amazônica.

PALAVRAS-CHAVE: floresta pluvial, morcego hematófago, Mato Grosso, Rondônia, Tocantins

CITE AS: Pedroso, M.A.; da Rocha, P.A.; Brandão, M.V.; Garbino, G.S.T.; de Moraes, C.O.; Aires, C.C. 2018. Filling gaps in the distribution of the whitewinged vampire bat, Diaemus youngii (Phyllostomidae, Desmodontinae): new records for southern Amazonia. Acta Amazonica 48: 154-157 
Bats of the subfamily Desmodontinae are very relevant to public health, as they are the only hematophagous mammals and major reservoirs and vectors of rabies virus (Johnson $e t$ al., 2014). The subfamily contains three species, Desmodus rotundus É. Geoffroy, 1810; Diphylla ecaudata Spix, 1823; and Diaemus youngii (Jentink, 1893) (Gardner 2008). Diaemus youngii has the fewest records in Brazil (Kwon and Gardner, 2008), being poorly known demographically and ecologically. The species is listed as "least concern" by the International Union for Conservation of Nature (IUCN) global assessment, mainly due to its wide geographic range (Aguiar et al., 2006; Greenhall and Schutt, 1996; IUCN, 2017). Diaemus youngii occurs over most of the Neotropical region, from northeastern Mexico, to Central and South America, reaching its southern limit in Misiones, Northern Argentina (Kwon and Gardner, 2008). In Brazil, the species is present in several phytophysiognomies, from Amazonian forests, through open habitats of the Pantanal, Cerrado and Caatinga biomes, to its southern limits in the Atlantic Forest of Paraná state (Brazil). Despite its wide distribution, records of the species are still rare and scattered (Greenhall and Schutt, 1996; Aguiar et al., 2006; Kwon and Gardner, 2008), especially in southern Amazonia, where a single record was known so far (Tavares et al., 2017). We report here the first record of D. youngii for Mato Grosso state, in central-western Brazil, and provide additional records for the states of Rondônia and Tocantins, in northern Brazil, extending the known distribution of the species in the southern Amazon (Figure 1).

All specimens analyzed here (Table 1) are deposited in the zoological collection of the Museu de Zoologia of Universidade de São Paulo (MZUSP). Its measurements are in accordance with the ones given in literature (see Greenhall and Schutt, 1996).

The three specimens were mist netted during bat inventories. On July 17, 2014, an adult male D. youngii (MZUSP 35712) (Figure 2A) from Abunã, district of Porto Velho, Rondônia state $\left(9^{\circ} 35^{\prime} \mathrm{S}, 65^{\circ} 3^{\prime} \mathrm{W}\right)$, was collected by the four main authors of the present study. The predominant vegetation type in that area is alluvial ombrophilous dense forest, with medium and large trees, palms, woody vines, and epiphytes (sensu Ivanauskas et al., 2008). The other two specimens refers to unpublished specimens from MZUSP collection. Specimen MZUSP 35713 (Figure 3), represented by a taxidermied skin and separated skull, was collected by Marília Kerr on April, 1997 at the Renato River, a tributary of the Teles Pires River, near the city of Cláudia (1 $11^{\circ} 24^{\prime} S$, $\left.55^{\circ} 2^{\prime} \mathrm{W}\right)$, state of Mato Grosso, Brazil. Further details on the

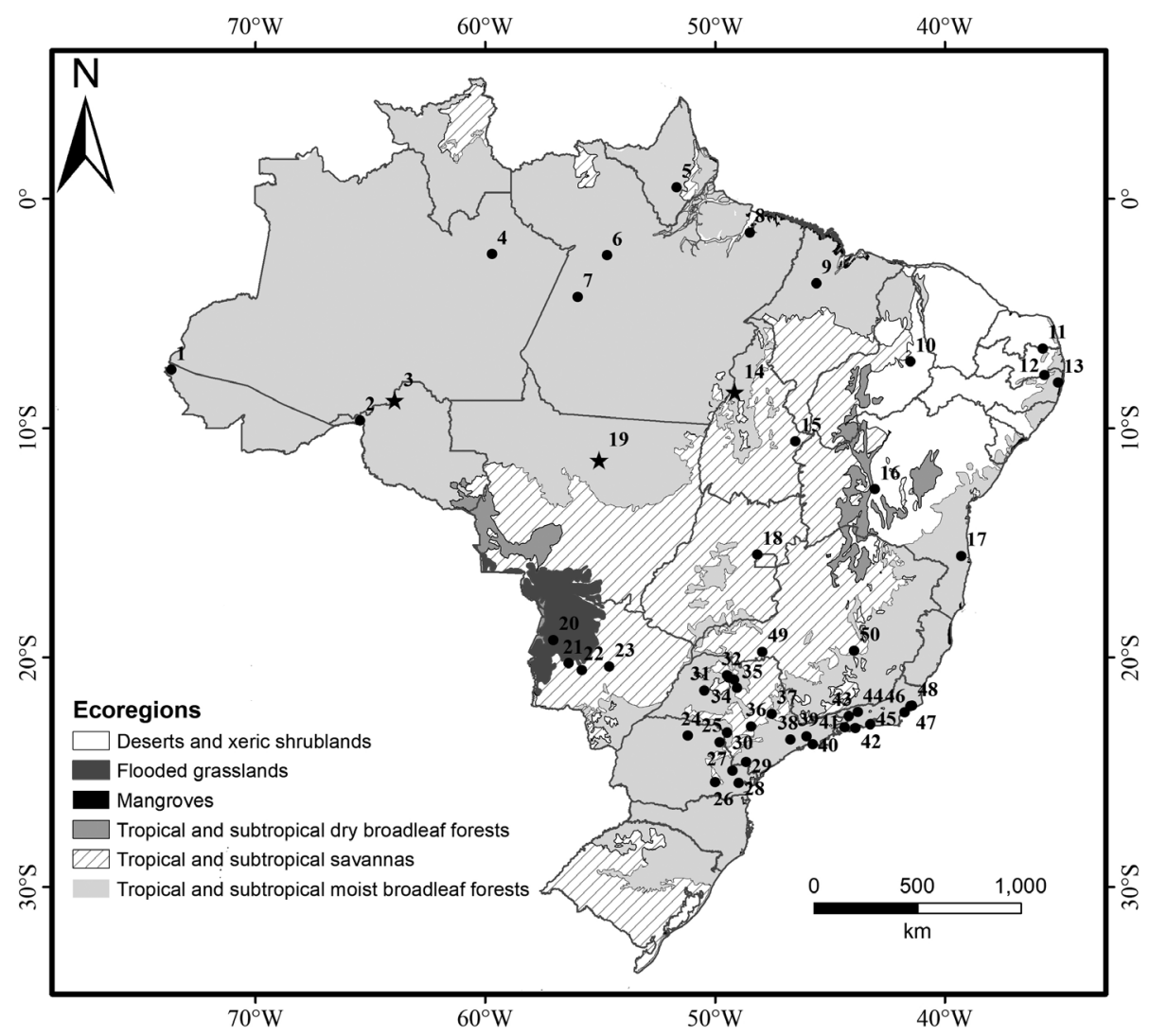

Figure 1. Known occurrence localities for Diaemus youngii. Stars represent the new records and circles are records from the literature. For key to code numbers, see Supplementary Material, Table S1. 
Table 1. Sex, external and cranial measurements (in $\mathrm{mm}$ ) of the Diaemus youngii specimens reported in here here. $\mathrm{RO}=$ Rondônia state, $\mathrm{MT}$ = Mato Grosso state, $\mathrm{TO}=$ Tocantins state.

\begin{tabular}{lccc}
\hline Parameter' & $\begin{array}{c}\text { MZUSP } \\
\text { 35712(RO) }\end{array}$ & $\begin{array}{c}\text { MZUSP } \\
35713(\mathrm{MT})\end{array}$ & $\begin{array}{c}\text { MZUSP } \\
35358(\mathrm{TO})\end{array}$ \\
\hline Sex & Male & Male & Male \\
\hline Body length & 76.46 & 71.15 & 73.61 \\
\hline Hind foot length & 15.35 & 16.94 & 16.40 \\
\hline Ear length & 17.49 & 16.06 & 18.05 \\
\hline Forearm length & 54.39 & 48.94 & 53.14 \\
\hline Greatest length of skull & 25.99 & 25.83 & 25.01 \\
\hline Condylobasal length & 21.59 & 20.35 & 21.09 \\
\hline Mastoid breadth & 13.20 & 11.84 & 12.69 \\
\hline Zygomatic breadth & 14.20 & 13.94 & 13.84 \\
\hline Breadth of braincase & 13.24 & 12.90 & 12.91 \\
\hline Postorbital constriction & 6.60 & 6.00 & 6.36 \\
\hline Palatal length & 8.08 & 7.82 & 7.61 \\
\hline Breadth across upp/er canines & 6.53 & 6.35 & 6.43 \\
\hline Breadth across upper molars & 6.80 & 6.53 & 6.41 \\
\hline Length of maxillary toothrow & 5.56 & 5.30 & 5.97 \\
\hline Length of mandible & 15.55 & 14.91 & 14.99 \\
\hline
\end{tabular}
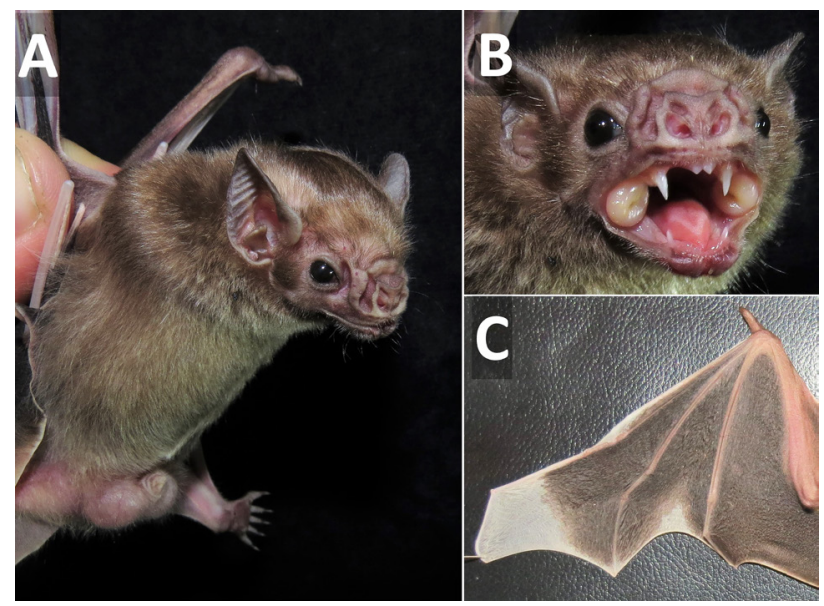

Figure 2. (A) Diaemus youngii (MZUSP 35712) caught in Porto Velho, state of Rondônia; (B) detail of the pair of glands located laterally on the inner part of the cheeks; (C) detail of the white spot on the distal tip of dactylopatagium. This figure is in color in the electronic version.

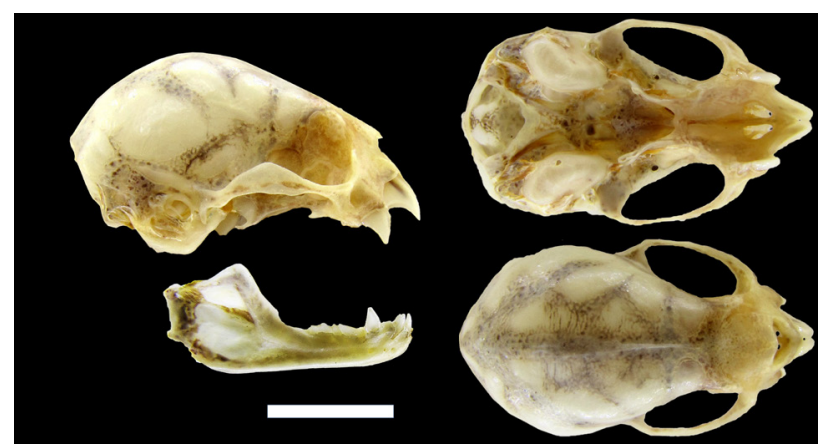

Figure 3. Dorsal, ventral and lateral views of the skull, and lateral view of the mandible of Diaemus youngii (MZUSP 35713) from the state of Mato Grosso, Brazil. Scale bar $=10 \mathrm{~mm}$. This figure is in color in the electronic version. latter collecting locality are available in Gualda-Barros et al. (2012). Specimen MZUSP 35358 (preserved in alcohol) was mist netted in Couto Magalhães ( $\left.8^{\circ} 21^{\prime} \mathrm{S}, 49^{\circ} 10^{\prime} \mathrm{W}\right)$, Tocantins state, in October 2014 during a bat inventory near an electric power transmission line (LT Xingu-Estreito) (Figure 1).

Diaemus youngii can be distinguished from the other two desmodontine genera by a suite of morphological characters: a pair of glands located laterally on the inner part of the cheeks (Figure 2B), that are exposed when the bat feels threatened and releases a strong-smelling liquid (Greenhall and Schutt, 1996); a white spot on the distal tip of the dactylopatagium and another spot between digits IV and V (Figure 2C); thumbs with only one basal pad, an absent calcar (in $D$. rotundus the calcar is present/, but greatly reduced, and in D. ecaudata it is conspicuous) (Kwon and Gardner, 2008); two upper and one lower molar (i1/2, c1/1, p1/2, m2/1=22) (Figure 3), while D. ecaudata has two lower molars (i2/2, $\mathrm{c} 1 / 1, \mathrm{p} 1 / 2, \mathrm{~m} 2 / 2=$ 26) and $D$. rotundus has one upper and one lower (i1/2, c1/1, $\mathrm{p} 1 / 2, \mathrm{~m} 1 / 1=20)$.

Our review of the records of $D$. youngii in Brazil show that records are concentrated in southeastern Brazil, in the states of São Paulo and Rio de Janeiro (Figure 1). This pattern is possibly a result of a collection bias, as in these two states intensive and long-duration surveys have been carried out, and consequently the bat fauna in the two regions is relatively better known (Esbérard and Bergallo, 2005; Garbino, 2016). Based on our review, there is a large sampling gap in the Cerrado of central Brazil, and in the interior Caatinga.

Roosts used by $D$. youngii include caves and cavities in trees (Aguiar et al., 2006; Greenhall and Schutt, 1996), however, contrary to D. ecaudata (Rocha et al., 2014) and D. rotundus (Oliveira et al., 2009; Greenhall et al., 1983; Flores-Crespo and Arellano-Sota, 1991; Bredt et al., 1999), the species is not commonly found in caves. Diaemus youngii has been found in caves in the southeastern Brazilian state of São Paulo, but with a low capture rate (Trajano, 1984), while in the same state, McNab (1969) and Taddei (Information on the specimen found in the Chiroptera Collection, Department of Zoology, State University of São Paulo, São José do Rio Preto "DZSJRP 16615”) sampled the species in cavities in standing trees. In Trinidad, a large colony was found in a hollow Erythrina micropteryx tree, and only a single individual was captured in a cave (Goodwin and Greenhall, 1961). Therefore, based on these data, it is suggested that additional records of $D$. youngii may be obtained by searching for roosts in hollow standing trees.

To have additional information on how and where to find the species, data on roost use and colony demographics are of special importance, expanding the knowledge of bloodfeeding bats and their biology. The records presented in here contribute to the knowledge on the distribution of D. youngii in the Amazon region. 


\section{ACKNOWLEDGEMENTS}

We are grateful to Arcadis Logos S/A for field campaigns and to Dr Mario de Vivo and Juliana Gualda de Barros from MZUSP, to CAPES for graduate stipend to MAP and for research stipend to PAR.

\section{REFERENCES}

Aguiar, L.M.S.; Camargo, W.R.; Portella, A.S. 2006. Ocurrence of white-winged vampire bat, Diaemus youngi (Mammalia, Chiroptera), in the Cerrado of Distrito Federal, Brazil. Revista Brasileira de Zoologia, 23: 893-896.

Bredt, A.; Uieda, W; Magalhães, E.D. 1999. Morcegos cavernícolas da regiáo do Distrito Federal, centro-oeste do Brasil (Mammalia, Chiroptera). Revista Brasileira de Zoologia, 16: 731-770.

Esbérard, C.E.; Bergallo, H.G. 2005. Research on bats in the state of Rio de Janeiro, southeastern Brazil. Mastozoologia Neotropical, 12: 237-243.

Flores-Crespo, R; Arellano-Sota, C. 1991. Biology and control of vampire bat. In: Baer, G.M. (Ed.). The Natural History of Rabies. CRC Press, Boca Raton, Flórida, p.462-474.

Garbino, G.S.T. 2016. Research on bats (Chiroptera) from the state of São Paulo, southeastern Brazil: annotated species list and bibliographic review. Arquivos de Zoologia, 47: 43-128.

Greenhall, A.M.; Joermann, G.; Schmidt, U. 1983. Desmodus rotundus. Mammalian Species, 202: 1-6.

Greenhall, A.M.; Schutt, Jr. W.A. 1996. Diaemus youngi. Mammalian Species, 533: 1-7.

Goodwin, G.G.; Greenhall, A.M. 1961. A review of the bats of Trinidad and Tobago: descriptions, rabies infection, and ecology. American Museum of Natural History, 122: 187-302.

Gualda-Barros, J.; Nascimento, F.O.; Amaral, M.K. 2012. A new species of Callicebus Thomas, 1903 (Primates, Pitheciidae) from the states of Mato Grosso and Pará, Brazil. Papéis Avulsos de Zoologia, 52: 261-279.

Ivanauskas, N.M.; Monteiro, M.; Rodrigues, R.R. 2008. Classificação fitogeográfica das florestas do Alto Rio Xingu. Acta Amazonica, 38: 387-402.

IUCN. 2017. The IUCN Red List of Threatened Species. Version $2017-$ 2. (http://www.iucnredlist.org). Accessed on 14 September 2017.

Johnson, N.; Aréchiga-Ceballos, N.; Aguilar-Setien, A. 2014. Vampire bat rabies: Ecology, epidemiology and control. Viruses, 6:1911-1928.
Kwon, M.; Gardner, A.L. 2008. Subfamily Desmodontinae. In: Gardner, A.L. (Ed.). Mammals of South merica, Volume 1, Marsupials, Xenarthrans, Shrews, and Bats. University of Chicago Press, Chicago, p.218-224.

McNab, B.K. 1969. The economics of temperature regulation in neutropical bats. Comparative Biochemistry and Physiology, 31: 227-268.

Oliveira, P.R.; Silva, D.A.R.; Rocha, J.H.; Melo, S.M.A.; Bombonato, N.G.; Carneiro e Silva, F.O. 2009. Levantamento, cadastramento e estimativa populacional das habitaçóes de morcegos hematófagos, antes e após atividades de controle, no município de araguari, Mg. Arquivos do Instituto de Biologia, 76: 553-560.

Rocha, P.A.; Pedroso, M.S.; Feijó, A.; Filho, N.G.; Bruno A.T.P. 2014. Update on the distribution of Diphylla ecaudata Spix, 1823 (Mammalia, Chiroptera): New records from the. Check List, 10: 1541-1545.

Tavares, V.C.; Nore, C.C.; Palmute, C.F.; Nogueira, E.P.P.; Gomes, J.D.; Marcos, M.H.; Silva, R.F.; Farias, S.G.; Bobrowiec, P.E.D. 2017. The bat fauna from southwestern Brazil and its affinities with the fauna of western Amazon. Acta Chiropterologica, 19: 93-106.

Trajano, E. 1984. Ecologia de populaçóes de morcegos cavernícolas em uma região cárstica do sudeste do Brasil. Revista Brasileira de Zoologia, 2: 255-320.

RECEIVED: $23 / 11 / 2017$

ACCEPTED: $19 / 02 / 2018$

ASSOCIATE EDITOR: Paulo Bobrowiec

\section{SUPPLEMENTARY MATERIAL}

(only available in the electronic version)

PEDROSO et al. Filling gaps in the distribution of the white-winged vampire bat, Diaemus youngii (Phyllostomidae, Desmodontinae): new records for southern Amazonia

Table S1. Locality records for Diaemus youngii in Brazil. The code numbers refer to the points shown in Figure 1. Datum: SAD69. 


\section{SUPPLEMENTARY MATERIAL (only available in the electronic version)}

PEDROSO et al. Filling gaps in the distribution of the white-winged vampire bat, Diaemus youngii (Phyllostomidae, Desmodontinae): new records for southern Amazonia

Table S1. Locality records for Diaemus youngii in Brazil. The code numbers refer to the points shown in Figure 1. Datum: SAD69.

\begin{tabular}{|c|c|c|c|c|c|}
\hline \multirow{2}{*}{ Code } & \multicolumn{2}{|c|}{ Coordinates } & \multirow{2}{*}{ State } & \multirow{2}{*}{ Locality } & \multirow{2}{*}{ Reference } \\
\hline & Lat & Long & & & \\
\hline 1 & $7027>00 » S$ & $73^{\circ} 41^{\prime} 00^{\prime \prime} \mathrm{W}$ & $A C$ & Parque Nacional da Serra do Divisor, Cruzeiro do Sul & Nogueira et al. (1999) \\
\hline 2 & 90 39'00"S & $65^{\circ} 27^{\prime} 00^{\prime \prime} \mathrm{W}$ & RO & Distrito de Abunã, Porto Velho & This study \\
\hline 3 & $8^{\circ} 48^{\prime} 06^{\prime \prime} S$ & $63^{\circ} 57^{\prime} 03^{\prime \prime} \mathrm{W}$ & RO & UHE Santo Antônio, Porto Velho & Tavares et al. (2017) \\
\hline 4 & $2^{\circ} 24^{\prime} 00^{\prime \prime} S$ & $59^{\circ} 43^{\prime} 00^{\prime \prime} \mathrm{W}$ & AM & Projeto Dinâmica Biológica de Fragmentos Florestais (PDBFF), Manaus & Bernard (2001) \\
\hline 5 & $0^{\circ} 30^{\prime} 00^{\prime \prime} \mathrm{S}$ & $51^{\circ} 40^{\prime} 00^{\prime \prime} \mathrm{W}$ & AP & Santa Luzia do Pacui, Macapá & Peracchi et al. (1984) \\
\hline 6 & $2^{\circ} 27^{\prime} 02^{\prime \prime} S$ & $54^{\circ} 42^{\prime} 03^{\prime \prime} \mathrm{W}$ & PA & Taperinha, Santarém & Piccinini (1974) \\
\hline 7 & $4^{\circ} 16^{\prime} 39^{\prime \prime} \mathrm{S}$ & $55^{\circ} 59^{\prime} 04^{\prime \prime} \mathrm{W}$ & PA & lataituba & Reis \& Schubart (1979) \\
\hline 8 & $1^{\circ} 27^{\prime} 21^{\prime \prime S}$ & $48^{\circ} 29^{\prime} 25^{\prime \prime} \mathrm{W}$ & PA & Utinga, Belém & Handley (1967) \\
\hline 9 & $3^{\circ} 41^{\prime} 00^{\prime \prime} S$ & $45^{\circ} 35^{\prime} 00^{\prime \prime} \mathrm{W}$ & MA & Tufilândia & Dias et al. (2009) \\
\hline 10 & $7004^{\prime} 54^{\prime \prime} S$ & $41^{\circ} 29^{\prime} 55^{\prime \prime} \mathrm{W}$ & PI & Picos & Pinto \& Bento (1986) \\
\hline 11 & $6^{\circ} 31^{\prime} 00^{\prime \prime} \mathrm{S}$ & $35^{\circ} 44^{\prime} 00^{\prime \prime} \mathrm{W}$ & PB & Parque Estadual Pedra da Boca, Araruna & Feijó et al. (2010) \\
\hline 12 & $7041^{\prime} 00^{\prime \prime} \mathrm{S}$ & $35^{\circ} 39^{\prime} 00^{\prime \prime} \mathrm{W}$ & PB & Lagoa da Pedra, Lajes, Umbuzeiro & Feijó \& Langguth (2011) \\
\hline 13 & $8^{\circ} 00^{\prime} 00^{\prime \prime} \mathrm{S}$ & $35^{\circ} 03^{\prime} 00^{\prime \prime} \mathrm{W}$ & PE & Estação Ecológica do Tapacurá, São Lourenço da Mata & Mares et al. (1981) \\
\hline 14 & $8^{\circ} 25^{\prime} 29^{\prime \prime} \mathrm{S}$ & $49^{\circ} 07^{\prime} 24^{\prime \prime} \mathrm{W}$ & TO & Estreito, Couto Magalhães & This study \\
\hline 15 & $10^{\circ} 34^{\prime} 00^{\prime \prime} \mathrm{S}$ & $46^{\circ} 30^{\prime} 00^{\prime \prime} \mathrm{W}$ & TO & Estação Ecológica Serra Geral do Tocantins & Gregorin et al. (2011) \\
\hline 16 & $12^{\circ} 39^{\prime} 00^{\prime \prime} \mathrm{S}$ & $43^{\circ} 03^{\prime} 00^{\prime \prime} \mathrm{W}$ & BA & Nas proximidades de Paratinga, Vale Médio do Rio São Francisco & Sá-Neto \& Marinho Filho (2013) \\
\hline 17 & $15^{\circ} 34^{\prime} 00^{\prime \prime} S$ & $39^{\circ} 17^{\prime} 00^{\prime \prime} \mathrm{W}$ & BA & Fazenda São José & Falcão (2007) \\
\hline 18 & $15^{\circ} 30^{\prime} 00^{\prime \prime} \mathrm{S}$ & $48^{\circ} 10^{\prime} 00^{\prime \prime} \mathrm{W}$ & DF & Brazlândia & Aguiar et al. (2006) \\
\hline 19 & $11^{\circ} 24^{\prime} 03^{\prime \prime} \mathrm{S}$ & $55^{\circ} 02^{\prime} 58^{\prime \prime} \mathrm{W}$ & MT & Cláudia & This study \\
\hline 20 & $19^{\circ} 13^{\prime} 10^{\prime \prime} \mathrm{S}$ & $57^{\circ} 02^{\prime} 30^{\prime \prime} \mathrm{W}$ & MS & Nhecolândia & Oliveira et al. (2012) \\
\hline 21 & $20^{\circ} 14^{\prime} 10^{\prime \prime} \mathrm{S}$ & $56^{\circ} 22^{\prime} 30^{\prime \prime} \mathrm{W}$ & MS & Miranda & Oliveira et al. (2012); Leite et al. (1998) \\
\hline 22 & $20^{\circ} 32^{\prime} 23^{\prime \prime} \mathrm{S}$ & $55^{\circ} 47^{\prime} 43^{\prime \prime} \mathrm{W}$ & MS & Aquidauana & Oliveira et al. (2012) \\
\hline 23 & $20^{\circ} 23^{\prime} 11^{\prime \prime} \mathrm{S}$ & $54^{\circ} 36^{\prime} 27^{\prime \prime} \mathrm{W}$ & MS & Instituto São Vicente, Campo Grande & Urbieta et al. (2017) \\
\hline 24 & $23^{\circ} 23^{\prime} 00^{\prime \prime} S$ & $51^{\circ} 11^{\prime} 00^{\prime \prime} \mathrm{W}$ & $P R$ & Parque Estadual Mata do Godoy & Reis et al. (2003) \\
\hline 25 & $23^{\circ} 40^{\prime} 25^{\prime \prime} \mathrm{S}$ & $49^{\circ} 47^{\prime} 36^{\prime \prime} \mathrm{W}$ & PR & Siqueira Campos & Margarido \& Braga (2004) \\
\hline 26 & $25^{\circ} 25^{\prime} 26^{\prime \prime} \mathrm{S}$ & $50^{\circ} 00^{\prime} 16^{\prime \prime} \mathrm{W}$ & $P R$ & Palmeira & Thomas (1899) \\
\hline 27 & $24^{\circ} 54^{\prime} 58^{\prime \prime} \mathrm{S}$ & $49^{\circ} 14^{\prime} 33^{\prime \prime} \mathrm{W}$ & $P R$ & Cerro Azul & Graciolli \& Carvalho (2001) \\
\hline 28 & $25^{\circ} 28^{\prime} 00^{\prime \prime} \mathrm{S}$ & $48^{\circ} 58^{\prime} 00^{\prime \prime} W$ & $P R$ & Estação Roça Nova, Piraquara & Miller (1906) \\
\hline 29 & $24^{\circ} 33^{\prime} 00^{\prime \prime} \mathrm{S}$ & $48^{\circ} 39^{\prime} 00^{\prime \prime} \mathrm{W}$ & SP & Caverna Alambari de Baixo, Iporanga & Trajano (1984) \\
\hline 30 & $23^{\circ} 15^{\prime} 27^{\prime \prime} \mathrm{S}$ & $49^{\circ} 28^{\prime} 01^{\prime \prime} \mathrm{W}$ & SP & Sarutaiá & Uieda (1993) \\
\hline 31 & $21^{\circ} 25^{\prime} 00^{\prime \prime} \mathrm{S}$ & $50^{\circ} 28^{\prime} 00^{\prime \prime} \mathrm{W}$ & SP & Bilac & Garbino (2016) \\
\hline 32 & $20^{\circ} 46^{\prime} 00^{\prime \prime} \mathrm{S}$ & $49^{\circ} 28^{\prime} 00^{\prime \prime} \mathrm{W}$ & SP & APA Grota de Mirassol, Mirassol & Garbino (2016) \\
\hline 33 & $20^{\circ} 52^{\prime} 00^{\prime \prime} \mathrm{S}$ & $49^{\circ} 24^{\prime} 00^{\prime \prime} \mathrm{W}$ & SP & Mata dos Macacos, São José do Rio Preto & Garbino (2016) \\
\hline 34 & $20^{\circ} 57^{\prime} 00^{\prime \prime} \mathrm{S}$ & $49^{\circ} 10^{\prime} 00^{\prime \prime} \mathrm{W}$ & SP & Uchoa & Garbino (2016) \\
\hline 35 & $21^{\circ} 19^{\prime} 00^{\prime \prime} \mathrm{S}$ & $49^{\circ} 03^{\prime} 00^{\prime \prime} \mathrm{W}$ & SP & Itajobi & Garbino (2016) \\
\hline 36 & $22^{\circ} 59^{\prime} 00^{\prime \prime} \mathrm{S}$ & $48^{\circ} 26^{\prime} 00^{\prime \prime} \mathrm{W}$ & SP & Botucatu & Uieda (2005) \\
\hline 37 & $22^{\circ} 27^{\prime} 00^{\prime \prime} S$ & $47032^{\prime} 00^{\prime \prime} \mathrm{W}$ & SP & Fazenda Paraguassu, Santa Gertrudes & Sazima \& Uieda (1980) \\
\hline 38 & $23^{\circ} 34^{\prime} 00^{\prime \prime} S$ & $46^{\circ} 43^{\prime} 00^{\prime \prime} \mathrm{W}$ & SP & Butantan, São Paulo & Vieira (1942) \\
\hline
\end{tabular}


Table S1. Continued

\begin{tabular}{|c|c|c|c|c|c|}
\hline \multirow{2}{*}{ Code } & \multicolumn{2}{|c|}{ Coordinates } & \multirow{2}{*}{ State } & \multirow{2}{*}{ Locality } & \multirow{2}{*}{ Reference } \\
\hline & Lat & Long & & & \\
\hline 39 & $23^{\circ} 25^{\prime} 00^{\prime \prime} \mathrm{S}$ & $46^{\circ} 01^{\prime} 00^{\prime \prime} \mathrm{W}$ & SP & Guararema & Garbino (2016) \\
\hline 40 & $23^{\circ} 46^{\prime} 00^{\prime \prime} S$ & $45^{\circ} 45^{\prime} 00^{\prime \prime} \mathrm{W}$ & SP & Barra do Uma, São Sebastião & Garbino (2016) \\
\hline 41 & $23^{\circ} 02^{\prime} 00^{\prime \prime} S$ & $44^{\circ} 21^{\prime} 00^{\prime \prime} \mathrm{W}$ & RJ & Ilha da Gipóia, Angra dos Reis & Carvalho et al. (2011) \\
\hline 42 & $23^{\circ} 04^{\prime} 00^{\prime \prime} S$ & $43^{\circ} 53^{\prime} 00^{\prime \prime} \mathrm{W}$ & RJ & Ilha de Marambaia, Rio de Janeiro & Lourenço et al. (2010) \\
\hline 43 & $22^{\circ} 32^{\prime} 00^{\prime \prime} S$ & $44^{\circ} 11^{\prime} 00^{\prime \prime} \mathrm{W}$ & RJ & Barra Mansa & Peracchi \& Albuquerque (1971) \\
\hline 44 & $22^{\circ} 22^{\prime} 00^{\prime \prime} S$ & $43^{\circ} 47^{\prime} 00^{\prime \prime} \mathrm{W}$ & RJ & Santuário da Vida Silvestre da Serra da Concórdia, Barra do Piraí & Modesto et al. (2008) \\
\hline 45 & $22^{\circ} 54^{\prime} 00^{\prime \prime} S$ & $43^{\circ} 14^{\prime} 00^{\prime \prime} \mathrm{W}$ & RJ & U.F.R.R.J., Seropédica & Peracchi \& Albuquerque (1984) \\
\hline 46 & $22^{\circ} 23^{\prime} 00^{\prime \prime} S$ & $41^{\circ} 45^{\prime} 00^{\prime \prime} \mathrm{W}$ & RJ & Parque Nacional da Restinga de Jurubatiba, Macaé & Luz et al. (2011) \\
\hline 47 & $22^{\circ} 07^{\prime} 00^{\prime \prime} S$ & $41^{\circ} 29^{\prime} 00^{\prime \prime} \mathrm{W}$ & RJ & Carmo & Avilla et al. (2001) \\
\hline 48 & $22^{\circ} 06^{\prime} 00^{\prime \prime} S$ & $41^{\circ} 25^{\prime} 00^{\prime \prime} \mathrm{W}$ & RJ & Parque Nacional Restinga de Jurubatiba, Quissamã & Bergallo et al. (2004) \\
\hline 49 & $19044^{\prime} 51^{\prime \prime} \mathrm{S}$ & $47056^{\prime} 21^{\prime \prime} \mathrm{W}$ & MG & Uberaba & Uieda (1993); Stutz et al. (2004) \\
\hline 50 & $19^{\circ} 42^{\prime} 00^{\prime \prime} S$ & $43^{\circ} 56^{\prime} 00^{\prime \prime} \mathrm{W}$ & MG & São José da Lapa & Torquetti et al. (2013) \\
\hline
\end{tabular}

Aguiar, L.M.S.; Camargo, W.R.; Portella, A.S. 2006. Ocurrence of white-winged vampire bat, Diaemus youngi (Mammalia, Chiroptera), in the Cerrado of Distrito Federal, Brazil. Revista Brasileira de Zoologia, 23: 893-896.

Avilla, L.S.; Rozensztranch, A.M.S.; Abrantes, E.A.L. 2001. First record of the South American Flat-Headed Bat Neoplatymops mattogrossensis (Vieira, 1942) in southeastern Brazil (Chiroptera, Molossidae). Boletim do Museu Nacional, 463: 1-6.

Bergallo, H.J.; Martins-Hatano, F.; Raíces, D.S.L.; Ribeiro, T.T.L.; Alves, A.G.; Luz, J. L.; Mangolin, R.; Mello, M.A.R. 2004. Os mamíferos da Restinga de Jurubatiba. In: Rocha, C.F.D.; Esteves, F.A.; Scarano, F.R. (Eds). Pesquisas de longa duração na Restinga de Jurubatiba-Ecologia, história natural e conservação. Rima Editora, São Carlos, São Paulo, p.215-230. Bernard, E. 2001. Vertical stratification of bat communities in primary forests of Central Amazon, Brazil. Journal of Tropical Ecology, 17: $115-126$.

Carvalho, W.D.; Freitas, L.N.; Freitas, G.P.; Luz, J.L.; Costa, L.M.; Esbérard, C.E.L. 2011. Efeito da chuva na captura de morcegos em uma ilha da costa sul do Rio de Janeiro, Brasil. Chiroptera Neotropical, 17: 808-816.

Dias, P.A.; Santos, C.L.C.; Rodrigues, F.S.; Rosa, L.C.; Lobato, K.S.; Rêbelo, J.M.M. 2009. Espécies de moscas ectoparasitas (Diptera, Hippoboscoidae) de morcegos (Mammalia, Chiroptera) no estado do Maranhão. Revista Brasileira de Entomologia, 53: 128-133.

Falcão, F.C. 2007. Mammalia, Chiroptera, Phyllostomidae, Diaemus youngi: First record for the state of Bahia, northeastern Brazil. Check List, 3: $330-332$.

Feijó, J.A.; Araújo, P.; Fracasso, M.P.A.; Santos, K.R.P. 2010. New records of three bats species for the Caatinga of the state of Paraíba, northeastern, Brazil. Chiroptera Neotropical, 16: 723-727.

Feijó, J.A.; Langguth, A. 2011. Lista de Quirópteros da Paraíba, Brasil com 25 novos registros. Chiroptera Neotropical, 17: $1055-1062$.

Garbino, G.S.T. 2016. Research on bats (Chiroptera) from the state of São Paulo, southeastern Brazil: annotated species list and bibliographic review. Arquivos de Zoologia, 47: 43-128.

Graciolli, G.; Carvalho, C.J.B. 2001. Mosca ectoparasita (Diptera, Hippoboscoidea) de morcegos (Mammalia, Chiroptera) do Estado do Paraná. II. Streblidae. Chave pictórica para gêneros e espécies. Revista Brasileira de Zoologia, 18: 907-960.

Gregorin, R.; Gonçalves, E.; Aires, C.C.; Carmignotto, A.P. 2011. Morcegos (Mammalia: Chiroptera) da Estação Ecológica Serra Geral do Tocantins: Composição específica e considerações taxonômicas. Biota Neotrop., 11: 298-311.

Handley Jr., C.O. 1967. Bats of the canopy of an Amazonian forest. Atas do simpósio sobre a biota Amazônica, 5: 211-15.

Lourenço, E.C.; Costa. L. M.; Silva, R.M.; Esbérard, C.E.L. 2010. Bat diversity of Ilha da Marambaia, Southern, Rio de Janeiro State, Brazil (Chiroptera, Mammalia). Braz. J. Biol., 70: 511-519.

Leite, A.P.; Meneghelli, M.; Taddei V.A. 1998. Morcegos (Chiroptera: Mammalia) dos pantanais de Aquidauana e da Nhecolândia, Mato Grosso do Sul. I. Diversidade de espécies. Ensaios e Ciência, 2: 167-174.

Luz, J.L.; Mangolin, R.; Ésberad, C.E.L.; Bergallo, H.G. 2011. Morcegos (Chiroptera) capturados em lagoas do Parque Nacional da Restinga de Jurubatiba, Rio de Janeiro, Brasil. Biota Neotrop., 11: 161-168.

Mares, M.A.; Willig, M.R.; Streilein, K.E.; Lacher, T.E. 1981. The mammals of northeastern Brazil: A preliminary assessment. Annals of the Carnegie Museum, $50: 81$-137.

Margarido, T.C.C.; Braga, F.C. 2004. Mamíferos. In: Mikich, S.B.; Bérnils, S. (Eds). Livro vermelho da fauna ameaçada no Estado do Paraná. Instituto Ambiental do Paraná, Curitiba, Paraná, p.27-142.

Miller Jr, G.S. 1906. Twelve new genera of bats. Proc. Biol. Soc., 19: 83-87.

Modesto, T.C.; Pessôa, F.S.; Jordão-Nogueira, T.; Enrici, M.C.; Costa, L.M; Attias, N.; Almeida, J.; Raíces, D.S.L.; Albuquerque, H.G.; Pereira, B.C.; Esbérard, C.L.; Bergallo, H.G. 2008. Mammals, Serra da Concórdia, state of Rio de Janeiro, Brazil. Check List, 4: 341-348.

Nogueira, M.R.; Pol, A.; Peracchi, A.L. 1999. New records of bats from Brazil with a list of additional species for the chiropteran fauna of the state Acre, western Amazon basin. Mammalia, 63: 363-368.

Oliveira, A.K.M.; Oliveira, M.D.; Favero, S.; Oliveira, L.F. 2012. Diversity, similarity and trophic guild of chiropterofauna in three southern Pantanal sub-regions, State of Mato Grosso do Sul, Brazil. Acta Scientiarum Biological Sciences, 34: 33-39.

Peracchi, A.L.; Albuquerque, T.S. 1971. Lista provisória dos quirópteros dos estados do Rio de Janeiro e Guanabara, Brasil. Rev.Bras. Biol., $31: 405-13$.

Peracchi, A.L.; Raimundo, S.D.L.; Tannure, A.M. 1984. Quirópteros do Território Federal do Amapá, Brasil (Mammalia, Chiroptera). Arq. Univ. Fed. Rural Rio de Janeiro, 7: 89-100.

Piccinini, R.S. 1974. Lista Provisória dos Quirópteros da Coleção do Museu Paraense Emílio Goeldi (Chiroptera). Boletim do Museu Paraense Emílio Goeldi, 77: 1-32.

Pinto, A.S.; Bento, D.N.C. 1986. Trypanossoma cruzi like bloodstream trypomastigotes in bats from the state of Piauí, northeastern Brazil. Revista da Sociedade Brasileira de Medicina Tropical, 19: 31-34.

Rei, N.R.; Schubart, H.O.R. 1979. Notas preliminares sobre os morcegos do Parque Nacional da Amazônia (médio Tapajós). Acta Amazonica, 9: 507-515

Reis, N.R.; Barbieri, M.L.; Lima, I.P.; Peracchi, A.L. 2003. What is better for maintaining the richness of bat (Mammalia, Chiroptera) species: A large forest fragment or many small fragments? Revista Brasileira de Zoologia, 20: 225-230.

Sá-Neto, R.J.; Marinho Filho, J. 2013. Bats in fragments of xeric woodland caatinga in Brazilian semiarid. Journal of Arid Environments, $90: 88-94$.

Sazima, I.; Uieda, W. 1980. Feeding behavior of the white-winged vampire bat, Diaemus youngii on poultry. J. Mammal., 61: 102-04.

Stutz, W.H.; Albuquerque, M.C.; Uieda, W.; Macedo, E.M.; Franca, C.B. 2004. Updated list of Uberlândia bats (Minas Gerais State, Southeastern Brazil). Chirop. Neotrop., 10: 188-190.

Tavares, V.C.; Nore, C.C.; Palmute, C.F.; Nogueira, E.P.P.; Gomes, J.D.; Marcos, M.H.; Silva, R.F.; Farias, S.G.; Bobrowiec, P.E.D. 2017. The bat fauna from southwestern Brazil and its affinities with the fauna of western Amazon. Acta Chiropterologica, 19: 93-106.

Thomas, O. 1899. List of the mammals obtained by Dr. Franco Grillo in the Province of Paraná, Brazil. Annal del Museo Civico di Storia Naturale, Genova, 2: 546-549.

Torquetti, C.G.; Assis, M.A.C.; Carmo, S.S.A.; Talamoni, S.A. 2013. Observations of a diurnal roost of the white-winged vampire bat Diaemus youngi in a karstic area of southeastern Brazil. Chiroptera Neotropical, 19: 1147-1150.

Trajano, E. 1984. Ecologia de populações de morcegos cavernícolas em uma região cárstica do sudeste do Brasil. Revista Brasileira de Zoologia, 2: $255-320$.

Uieda, W. 1993. Comportamento alimentar do morcego hematófago Diaemus youngi, em aves domésticas. Revista Brasileira de Biologia, 53: $529-538$.

Uieda, W. 2005. Bats from Botucatu region, state of São Paulo, southeastern Brazil. Chiroptera Neotropical, 11: 224-226.

Urbieta, G.L.; Siqueira, T.Y.S.; Graciolli, G. 2017. White-winged Vampire Bat, Diaemus youngi (Jentink, 1893) (Mammalia, Chiroptera): range extension in the Cerrado biome and new locality in Mato Grosso do Sul, southwestern Brazil. Check List, 13: 2128.

Vieira, C.O.C. 1942. Ensaio monográfico sobre os quirópteros do Brasil. Arq. Zool, 3: 219-471. 